\title{
Hybrid Petri nets with general one-shot transitions for dependability evaluation of fluid critical infrastructures
}

\author{
Marco Gribaudo \\ Dipartimento di Elettronica e Informazione \\ Politecnico di Milano, Italy \\ marco.gribaudo@di.unito.it
}

\author{
Anne Remke \\ Design and Analysis of Communication Systems \\ University of Twente, The Netherlands \\ a.k.i.remke@utwente.nl
}

\begin{abstract}
A hybrid Petri net formalism that is specifically tailored towards so-called fluid critical infrastructures is introduced, allowing for timed, generally distributed and fluid transitions. Such models are analyzed with Parametric Reachability Analysis, by separating the deterministic and the stochastic evolution of the system. Several performance metrics, such as the distribution of fluid over time, can be derived by deconditioning according to arbitrary continuous probability distributions. This efficient concept allows for the analysis of an arbitrary number of fluid places, as opposed to classical stochastic hybrid Petri net approaches. Moreover, validation of our results against the FSPN tool shows that parametric reachability analysis provides more accurate results. A case study motivates and shows the feasibility of our approach.
\end{abstract}

\section{INTRODUCTION}

More and more our society and economy rely on the welloperation of fluid critical infrastructures [1], [2], such as water treatment and distribution networks. Such systems are characterized by deterministic fluid transportation, however, with rates that change according to a stochastic process.

Hence, Fluid Stochastic Petri Nets (FSPNs)[3], [4], [5] and Piece-wise Deterministic Markov Processes (PDMPs) [6] appear to be suitable. However, the memory of continuous variables in PDMPs is lost upon stochastic transitions. Hence, they cannot model the physical behaviour of fluid critical infrastructures. FSPNs have a clear mathematical background that allows to write equations that completely characterize the evolution of any FSPN model. However, in general such equations can be solved only when there are at most one or two continuous variables. Simulation is the only available alternative when considering larger models, and also in those cases simulation is not a trivial task [7], [8]. Hybrid Petri nets, as introduced by David and Alla [9], can be mapped to hybrid automata [10] if no stochastic behavior is considered. However, hybrid Petri nets with a stochastic discrete part suffer from the same restrictions as FSPNs.

This clearly shows the need for a modeling and analysis framework that is specifically tailored towards fluid critical infrastructures. We exploit the fact that the system under study is fairly deterministic. Only failure and repair events are stochastic, hence, there are relatively few stochastic transitions. This allows separating the deterministic and the stochastic evolution of the system, using a conditioning / deconditioning argument. This speeds up the reachability analysis and allows for a large number of continuous variables in the model, as opposed to previous approaches.

This paper introduces a hybrid Petri net formalism with General transitions (HPNG). We allow an arbitrary number of continuous places that can be connected via fluid transitions. These transitions are controlled by discrete places that can be connected via deterministic and generally distributed transitions. Generally distributed transitions must respect the constraint that they can fire only once during the evolution of the model: for this reason we address them as oneshot transitions. Moreover, test and inhibitor arcs allow to condition on the amount of tokens in discrete places.

We introduce a new and efficient concept for the computation of all reachable locations of a model: Parametric reachability analysis. This technique separates the deterministic and the stochastic components of a HPNG by conditioning the deterministic evolution on the samples drawn from the probability distributions associated to general transitions. After all reachable parametric locations have been computed, several important performance metrics (such as the distribution of fluid over time) can be derived by deconditioning, i.e., by integrating over the values of the probability distributions that characterize the general transitions. In this work we concentrate only on systems characterized by a single general transition. However, the proposed formalism can be extended to cases with more than one generally distributed transition. As opposed to similar SHM solution algorithms, our technique is not affected by the number of fluid places, which can be arbitrary.

A HPNG with one fluid place and one exponentially distributed general transition is in fact a FSPN that can be solved using numerical techniques. We exploit this fact to validate our approach by comparing the solutions obtained using the parametric reachability analysis and an existing FSPN tool. As we will see, the results provided by parametric reachability analysis are more accurate than those obtained with FSPN techniques. The concept of parametric reachability analysis as proposed in this paper resembles the Reachable state-classes presented in [11]. However, that 
work does not take into account continuous variables: if we put our formalism in their framework, we obtain nonstochastic locations that depend on the value of the stochastic variables and that are not necessarily of DBM (Difference Bounds Matrix) type, destroying thus the fundamental hypothesis of their approach. The proposed methodology can be used to analyze the dependability of fluid critical infrastructures, that require discrete and continuous variables. As a case study, we present a HPNG model of the last phases of a water treatment facility, with different demand during nights and days.

This paper is further organized as follows: Section II introduces the modeling formalism of hybrid Petri nets with General one-shot transitions. The parametric reachability algorithm is presented and illustrated on a small example in Section III. The different measures of interest that can be computed are discussed in Section IV, including a validation of the results. Section V shows the feasibility of the approach with a case study on a water cleaning facility before we conclude in Section VI.

\section{HPNGS: MODEL DEFINITION}

We introduce the model definition for a hybrid Petri net formalism with a discrete part that closely resembles GSPNs [12] and a continuous part that closely resembles Hybrid Petri Nets [9]. We generalize the neg. exponentially distributed stochastic transitions of GSPNs to arbitrary distributed stochastic transitions and extend the concept of immediate transitions to deterministic transitions that fire after a fixed delay. Due to the restricted amount of space we cannot describe the model syntax and evolution in all detail. For this we refer to [13].

A hybrid Petri net with general one-shot transitions is defined as a tuple $\left(\mathcal{P}, \mathcal{T}, \mathcal{A}, \mathbf{M}_{0}, \Phi\right)$. Here $\mathcal{P}=\mathcal{P}_{D} \cup \mathcal{P}_{F}$ is a finite set of places that holds both, discrete and continuous places. A discrete place $P \in \mathcal{P}_{D}$ contains a natural number of tokens $m_{P} \in \mathbb{N}$, whereas a fluid place $C \in \mathcal{P}_{F}$ contains a non-negative level of fluid $n_{C} \in \mathbb{R}_{0}^{+}$. The initial marking $\mathbf{M}_{0}=\left(\mathbf{m}_{0}, \mathbf{n}_{0}\right)$ then describes the initial amount of tokens in all discrete places and the initial amount of fluid in all continuous places. The finite set of transitions $\mathcal{T}=\mathcal{T}_{I} \cup \mathcal{T}_{T} \cup \mathcal{T}_{G} \cup \mathcal{T}_{F}$ is composed of the set of immediate transitions, the set of deterministically timed transitions, the set of generally distributed transitions and the set of fluid transitions. Note that in this paper we restrict the number of general transitions to $\left|\mathcal{T}_{G}\right|=1$. The set of $\operatorname{arcs} \mathcal{A}$ is divided into four sets, as follows. The set of discrete input and output $\operatorname{arcs} \mathcal{A}_{D}$, connects discrete places and discrete transitions and the set of fluid input and output arcs $\mathcal{A}_{F}$ connects fluid places and fluid transitions. The set of inhibitor arcs $\mathcal{A}_{I}$ and the set of test arcs $\mathcal{A}_{T}$, both connect discrete places to all kinds of transitions. Note that, currently, every fluid transitions can only have one input and one output arc.

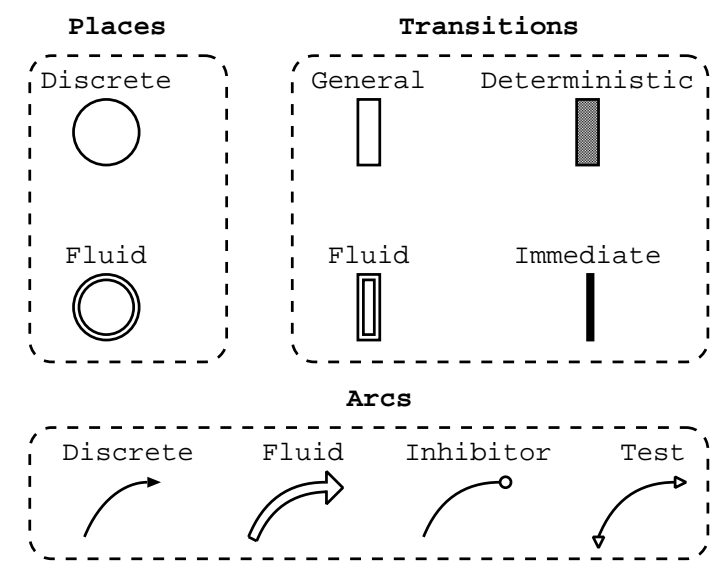

Figure 1. Primitives for the hybrid Petri net formalism with general oneshot transitions

The tuple $\Phi$ contains 9 parameter functions $\Phi=$ $\left(\phi_{b}^{P}, \phi_{w}^{T}, \phi_{p}^{T}, \phi_{d}^{T}, \phi_{f}^{T}, \phi_{g}^{T}, \phi_{w}^{A}, \phi_{s}^{A}, \phi_{p}^{A}\right)$. Here $\phi_{b}^{P}: \mathcal{P}_{F} \rightarrow$ $\mathbb{R}^{+} \cup \infty$ defines an upper bound per fluid place. In case of $\phi_{b}^{P}(p)=\infty$, the place can hold an unbounded amount of fluid. Per transition, the weight is defined by $\phi_{w}^{T}: \mathcal{T} \backslash \mathcal{T}_{F} \rightarrow$ $\mathbb{R}^{+}$and the priority by $\phi_{p}^{T}: \mathcal{T} \backslash \mathcal{T}_{F} \rightarrow \mathbb{N}$. The weight and priority that is associated to all but fluid transitions is used to resolve firing conflicts of transitions that are supposed to fire at the same time instant [12]. Only transitions with the highest priority fire with a probability according to their weight. All other transitions fire with probability zero. For a timed transition, the deterministic firing time is constant and defined by $\phi_{d}^{T}: \mathcal{T}_{D} \rightarrow \mathbb{R}^{+}$, for a fluid transition the nominal firing speed is a constant and defined by $\phi_{f}^{T}: \mathcal{T}_{F} \rightarrow \mathbb{R}^{+}$. A continuous density function is assigned to the general transition by $\phi_{g}^{T}: \mathcal{T}_{G} \rightarrow\left(p: \mathbb{R}^{+} \rightarrow[0,1]\right)$. Weight is assigned to all but fluid arcs: $\phi_{w}^{A}: \mathcal{A} \backslash \mathcal{A}_{F} \rightarrow \mathbb{N}$ and defines the amount of tokens that is taken from or added to connected places upon firing of the transition.

Conflicts in the distribution of fluid occur when a fluid place reaches one of its boundaries. To prevent overflow, the fluid input has to be reduced to match the output, and to prevent underflow the fluid output has to be reduced to match the input, respectively. The firing rate of fluid transitions is then adapted according to the share $\phi_{s}^{A}: \mathcal{A}_{F} \rightarrow \mathbb{R}^{+}$ and priority $\phi_{p}^{A}: \mathcal{A}_{F} \rightarrow \mathbb{N}$ that is assigned to the fluid arcs that connect the transition to the place. This is done by distributing the available fluid over all fluid arcs. Those with highest priority are considered first and if their is enough fluid available, all transitions with the highest priority can still fire at their nominal speed. Otherwise, their fluid rates are adapted according to the firing rate of the connected transitions and the share of the arc, according to [9]. The adaptation of fluid rates in these cases, results in a piecewise constant fluid derivative per fluid place. 
Graphical representation: The primitives of the hybrid Petri net formalism with general one-shot transitions are shown in Figure 1. A discrete place is graphically represented by a single circle and a fluid place is represented by two concentric circles. A general transition is drawn as an empty rectangle, a deterministic transition is drawn as a grey rectangle, a fluid transitions shown as an empty rectangle with double lines and an immediate transition is a thick black bar. The discrete input and output arcs are drawn as single arrows and fluid input and output arcs are represented with double lines. Inhibitor arcs are drawn with a small circle toward the transition and test arcs are drawn with two triangular arrowheads .

\section{PARAMETRIC REACHABILITY ANALYSIS}

The set of all the reachable parametric locations can be computed by separating the deterministic and the stochastic evolution of the system. This can be accomplished by conditioning the deterministic evolution on the value of a sample of the probability distribution associated to the general transition. Starting from the initial parametric location the main algorithm for the computation of the next location and the firing of an event is discussed. Furthermore, the algorithms for the derivation of the next minimum event before and after the firing of the general transition is introduced. The concept is explained and visualized on a small example.

Parametric location: To describe the deterministic evolution of the system conditioned on the sample of the general probability distribution, an overall clock $\tau \in \mathbb{R}^{+}$ that maintains the evolution of time is needed. Then, in addition to the vectors $\mathbf{m}$ and $\mathbf{n}$, describing the number of tokens in the discrete states and the amount of fluid in the continuous states, a vector $\mathbf{n}^{\prime}$ represents the derivative with which the fluid changes over time per place. Furthermore, we add a vector of clocks $\mathbf{x}$ representing the remaining time to fire for every deterministic transition and a clock $g \in \mathbb{R}^{+} \cup\{-1\}$ which represents the time where the general transition was disabled, if it has not fired yet, or which equals -1 if it has fired already. Furthermore, the interval $[l, r]$ with $l, r \in \mathbb{R}^{+} \cup \infty$ and $l \leq r$ indicates the range of possible values for the sample of the probability distribution. Finally, we add the probability $p$ to reach the parametric location at time $x$.

The tuple $\mathbf{L}=\left(\tau, \mathbf{m}, \mathbf{n}, \mathbf{n}^{\prime}, \mathbf{x}, g,[l, r], p\right.$, next $)$ then completely describes a state that the Petri net enters at time $z$ and is called a parametric location. The initial parametric location is initialized as follows: the evolution time is set to zero and the derivative of the fluid places is set such that the influx equals the outflow of fluid places that are at the boundaries, based on the priority, share and flow rate of the enabled fluid transitions, as in [9]. For every deterministic transition $t \in \mathcal{T}_{D}$ the clock $x_{t}$ and the clock of the general transition $g$ are set to zero, $[l, r]$ is initialized to $[0, \infty]$ and $p$ is set to 1 . Starting from the initial marking $\mathbf{M}_{0}$, Algorithm 1 computes the state-space of the parametric locations until time $\tau_{\max }$ is reached (line 3 ) or all locations are absorbing, i.e., there are no more locations to visit.

Computing the next location: In case immediate transitions are enabled (line 4) no other transition types need to be considered and the immediate transitions with the highest priority are fired according to Algorithm 2. A new parametric location is reached with the firing of each immediate transition and the algorithm is called recursively for each new location. Algorithm 1, line 9, represents the case where no immediate transitions are enabled and the general transition has not fired yet. In this case the next location is either reached by the firing of the general transition or by a deterministically scheduled event. Before the firing of the general transition the next minimum event time $\tau_{\min }$ is unique; however several deterministic events with the same priority can be scheduled at the same time. In line 10, of Algorithm 1 the function compNextMin (loc) is called, which computes the relative time $\tau_{\min }$ after which the next deterministic event will fire and the set of events that happen at time $\tau_{\min }$, according to Algorithm 3 .

With the firing of the general transition in Algorithm 1, line 12 a new parametric location is reached and the general transition is scheduled to fire before $\tau_{\min }$ elapses. Hence, the sample of the probability distribution $s$ has to be smaller than the absolute firing time of the next deterministic event $\tau+\tau_{\text {min }}$ minus the time the general transition has been disabled before $g$. The right bound of the interval is set accordingly in line 13 while $l$ remains unchanged. The current time now equals the sample of the general transition $s$ plus the disabling time $g$ and then $g$ is set to -1 to indicate that the general transition has fired. Then the algorithm is called recursively for the new location. From now on, the current time, the fluid marking and the clocks of the deterministic transitions may depend linearly on the value of $s$. Hence, $\tau, \mathbf{n}$ and $\mathbf{x}$ must be seen as functions of $s$. However for readability reasons we keep the notation as above. From line 18 on the deterministic event(s) are scheduled before the firing of the general transition. With the firing of each of these events a new parametric location is reached. If the general transition is enabled the left interval is increased to $\tau+\tau_{\min }-g$ in line 21 . Otherwise, the interval remains unchanged but the $\tau_{\min }$ has to be added to the disabling time, as in line 23 . In any case, the time is increased by $\tau_{\min }$ and the algorithm is called for the new location. After the general transition has fired (starting from line 28), the time at which events fire may depend linearly on the value of $s$ and can hence be seen as a line. In line 29 function createListLine ( $\mathrm{LOC})$ is called, which returns a list of lines that correspond to the deterministic transitions with the highest priority and all fluid places with a non-zero derivative, according to Algorithm 4. For different parts of the current interval $[l, r]$ different events may befirst, depending on the slope of the lines and their intersection 


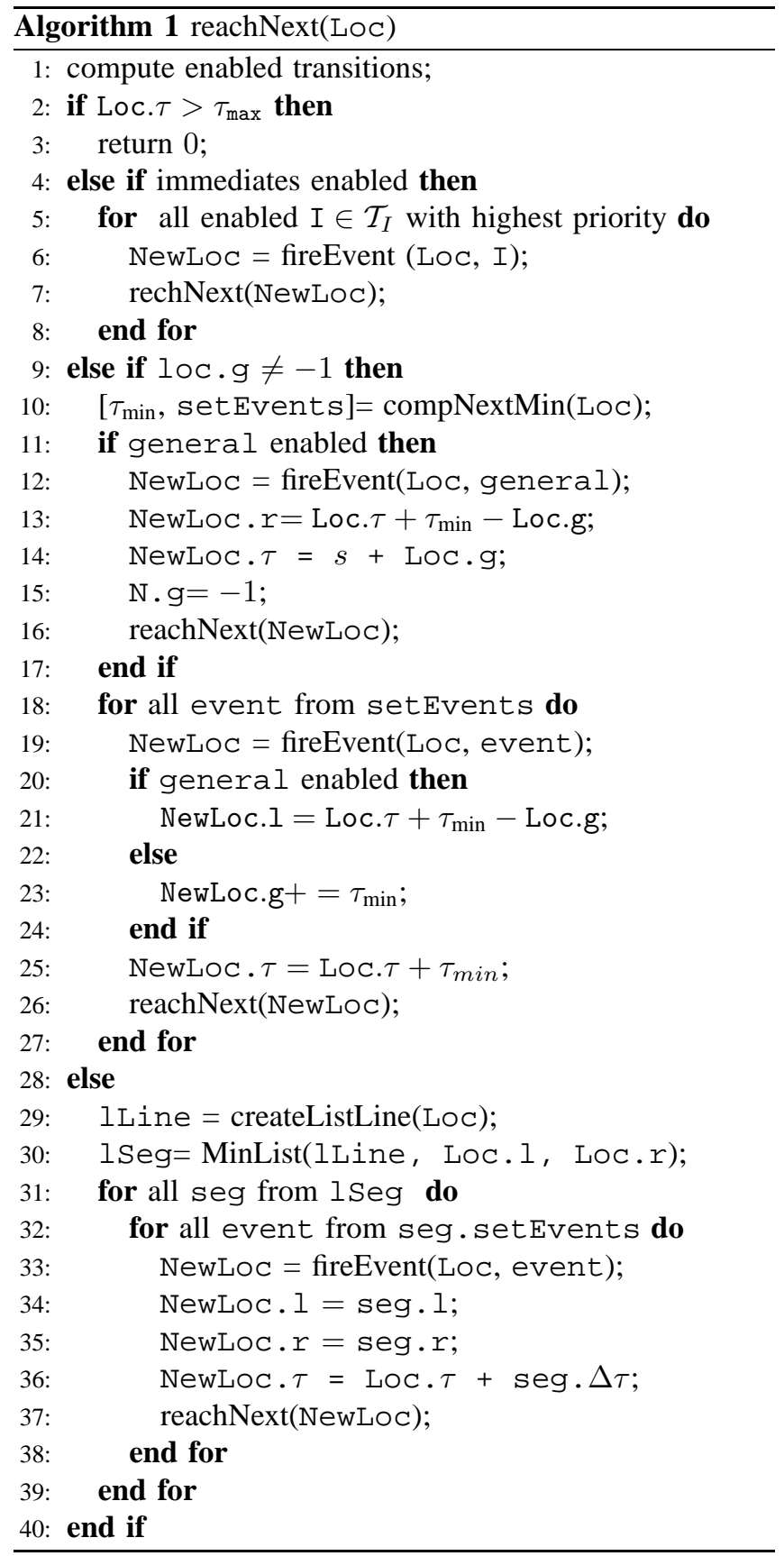

points. In line 30, function MinList (lLine, Loc.1, LOC.r) is called which computes a list of segments. Each segment contains a set of minimum events setEvents that fire after $\tau_{\min }$ between two intersection points [seg.l, seg.r], according to Algorithm 5. For every segment of the list then with the firing of all events that belong to the minimum set of events in line 33 a new parametric location is reached. The interval and the time are updated accordingly and the algorithm is called recursively for the new location.

Firing of an event: A new parametric location is reached with the firing of each immediate transition, as presented in Algorithm 2. First the values of the starting location are copied to the new location and the new location is added to the graph (line 1). If the event that is fired is an immediate transition, only the discrete marking has to be updated, as the fluid levels and the clocks do not change in zero time. However, the probability has to be updated to its weight divided by the weight of all enabled immediate transitions with corresponding priority. If the event is a general transition, the discrete and the fluid marking, the derivatives and the clocks are updated, according to line 5. For a deterministic transition, the complete marking, the derivatives of the fluid places and the clocks have to be updated. The probability is set to the weight of the deterministic transition divided by the weight of all enabled deterministic transitions with corresponding priority. In case the event is the reaching of a fluid bound, only the continuous marking, the derivatives of the fluid places and the clocks have to be updated.

Next minimum event before the general transition: In case the general transition has not fired yet, Algorithm 3 is used to compute the set of next minimum deterministic events. For all enabled transitions with the highest priority the remaining time to fire is compared. In case a new minimum time to fire was found, $\tau_{\min }$ is reset and the set is changed to the set with only the corresponding transition (line 6). If another transition with the same minimum time to fire is found, it is added to the set (line 8). After the relevant timed transitions have been considered, all fluid places with non-zero drift have to be checked. For all fluid places with positive drift, the time until the reaching of the upper bound has to be checked. This is given by $\left(\phi_{b}^{P}(C)-\right.$ Loc. $\left.\mathrm{n}_{\mathrm{C}}\right) /$ Loc. $\mathrm{n}_{\mathrm{C}}^{\prime}$. Only if this time is smaller than the minimum time to fire of a timed transition the fluid place has to be explicitly considered (line 13). If the time to reaching the bound equals the current $\tau_{\min }$ the fluid will be automatically updated when the deterministic transition fires and hence, it does not have to be taken into account. For fluid places with negative drift, the time to reaching the lower bound Loc. $\mathrm{n}_{\mathrm{C}} / \mid$ Loc. $\mathrm{n}_{\mathrm{C}}^{\prime} \mid$ is checked, respectively, from line 17 onwards.

Next minimum events after the general transition: As pointed out, after the firing of the general transition the time, the clocks and the fluid level are all linear functions of $s$. Algorithm 4 returns a list of the events that have to be considered to find the next minimum event. For all enabled timed transitions with the highest occurring priority the remaining time of transition $T$ is given by the deterministic time to fire $\phi_{d}^{T}(T)$ minus the time that has elapsed since the transition became enabled $X_{T}$ (line 3 ). For a fluid places $C^{+}$with a positive derivative, the remaining time until the upper bound is reached is given by the upper bound $\phi_{b}^{P}\left(C^{+}\right)$minus the current fluid level $\mathbf{n}_{C^{+}}$divided by the derivative $\mathbf{n}_{C^{+}}^{\prime}$ (line 7 ). In a similar way the remaining time to fire for place $C^{-}$with a negative derivative, is computed 

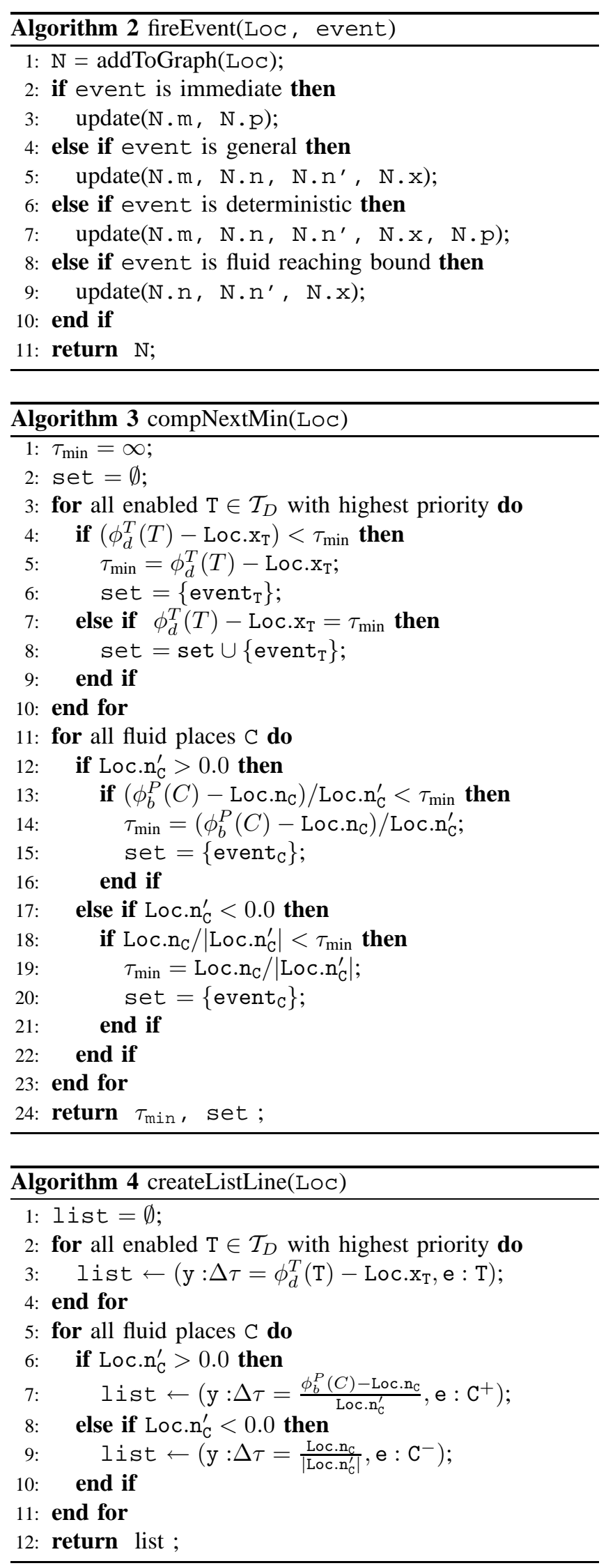

considering that the lower bound is always 0 (line 9 ). The list of lines that is returned by Algorithm 4 is then used as input for Algorithm 5 which computes the intersection points of all the lines that partition the interval $[l, r]$ into segments and returns the minimum event(s) between segment. In the following notation $y(l)$ is used to denote the value of function $y$ at $l$. Starting at the left bound of the interval $[l, r]$, the lowest line(s) have to be found, as they represent the minimum next event. Setting the minimum line to the first line from the list at line 2 , the value of each other line at the left interval $l$ is compared with the current minimum line at $l$. In case a line is found that returns a smaller value at the left interval, it is denoted the new minimum (line 5). If a line with the same value at $l$ but a smaller slope is found

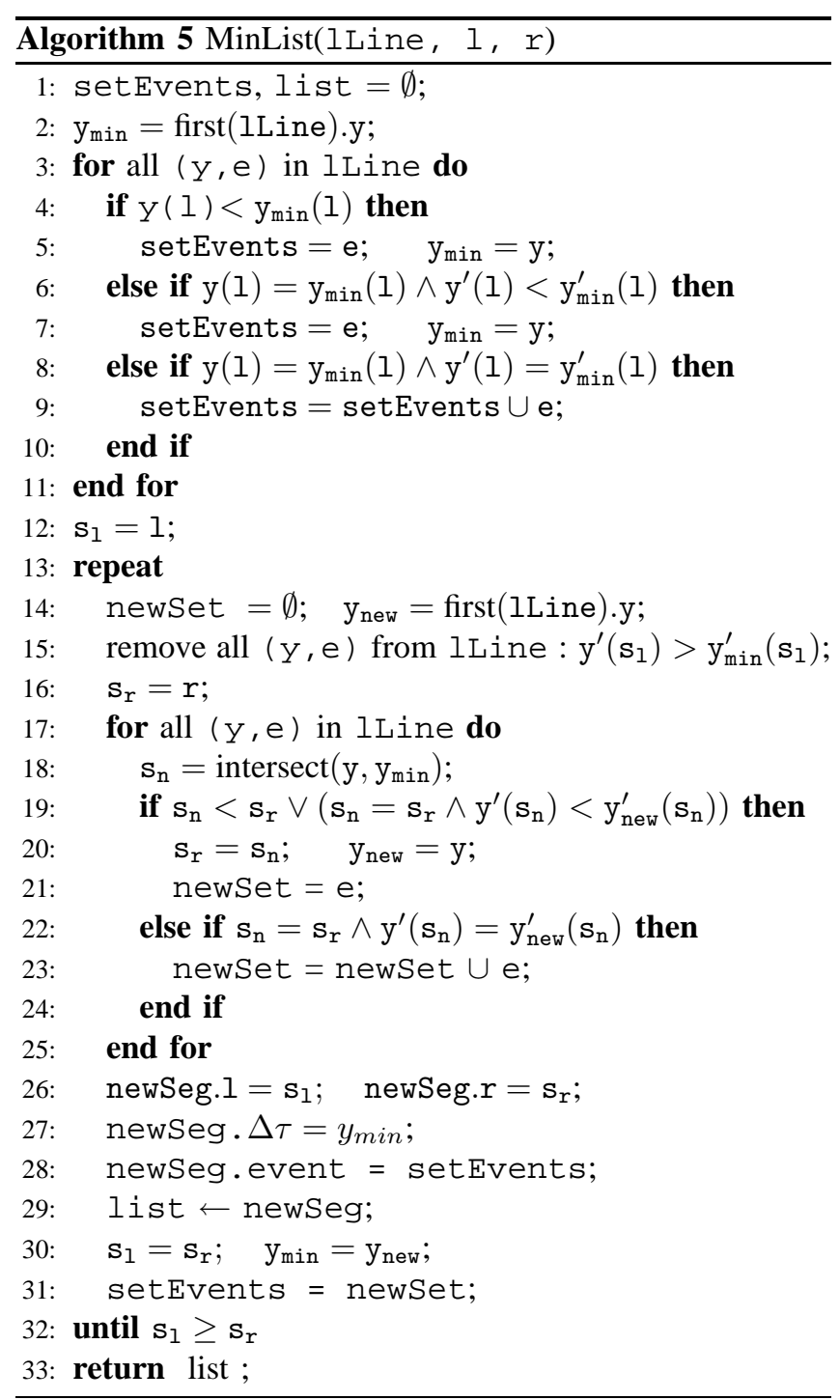

(line 6), it is also denoted the new minimum. However, in case a line with the same value at $l$ and the same slope is found, it has to be added to the set of minimum events, as 
we have found two events with the same minimum function.

Then from line 13 on, repeatedly the next intersection point with the current set of minimum lines has to be found and the new set of minimum lines, after the intersection point has to be computed. After initializing the necessary extra variables in line 14, all lines with a larger slope are removed from input set of lines lLine in line 15. From the remaining lines, the one with the closest intersection point and the smallest slope forms the new set of minimum events, starting from line 17 . In case a line has the same minimum intersection point and the same slope, it is added to the new set of minimum events (line 23). The new segment is then formed by the current intersection point $s_{l}$ and the intersection point that has just been found $s_{r}$. The remaining time to fire $\Delta \tau$ is given by the minimum line $y_{\min }$ and the set of events contains all events that have the same minimum time to fire. The new segment is added to the list of segments (line 29) and the values of the variables are swapped such the new intersection point becomes the current intersection point. This is repeated until the newly found intersection point is greater or equal to the right interval $r$.

Reservoir example: In the following we illustrate the parametric reachability analysis for the example hybrid Petri net, given in Figure 2. It models a reservoir with deterministically timed demand $(\mathrm{T}=5)$ and a generally distributed pump breaking time. As long as a token is present in the discrete place $P_{p}$, the pump $F_{p}$ is working and pumps fluid into the reservoir (continuous place $C_{r}$ ) with rate $R=2$. While place $P_{d}$ has a token, the demand $F_{d}$ is on and takes fluid from the reservoir at rate $R=1$. The reservoir can hold between 0 and 10 units of fluid.

The reachable parametric locations are shown in Figure 3. Starting in location 0 the minimum next deterministic event is the firing of $T_{e}$ at $\tau_{\min }=5$, as the filling of the fluid place with $n_{r}^{\prime}=1$ will take 10 time units. Hence, location 5 is reached by firing $T_{e}$ and conditioning $s \in[5, \infty]$. Location 1 is reached if $G_{b}$ fires first. In this case the sample $s$ of the probability distribution is conditioned to be $s \in[0,5]$. For both new locations the discrete and fluid marking, the value of the clocks and the time have to be updated. In location 1 the time $\tau=s$, the fluid level $n_{r}=s$ and the clock $x_{e}=s$ depend linearly on $s$. The next minimum deterministic event is either the firing of transition $T_{e}$ at $s+\Delta \tau=5$ or the fluid place reaching its lower boundary at $s-\Delta \tau=0$. As illustrated in Figure 4 for $s \in[0,2.5]$ the next minimum event will be the reaching of the lower bound at place $C_{r}$, while for $s \in[2.5,5]$ the next event is the firing of transition $T_{d}$. Hence, from location 1 the two new parametric locations 2 and 4 are reachable, depending on the value of $s$. In this example, the underlying state space consists of 9 parametric locations, where location 0 is the initial location and the parametric locations $3,4,6,7$, and 8 are absorbing, as they cannot be left anymore.

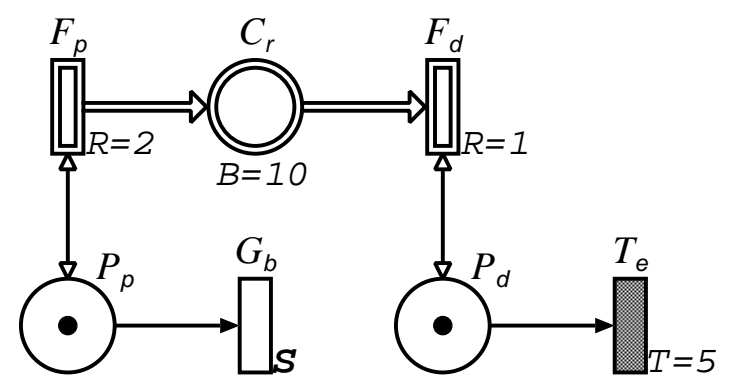

Figure 2. Hybrid Petri et model of a reservoir with deterministically timed demand and a generally distributed pump breaking time

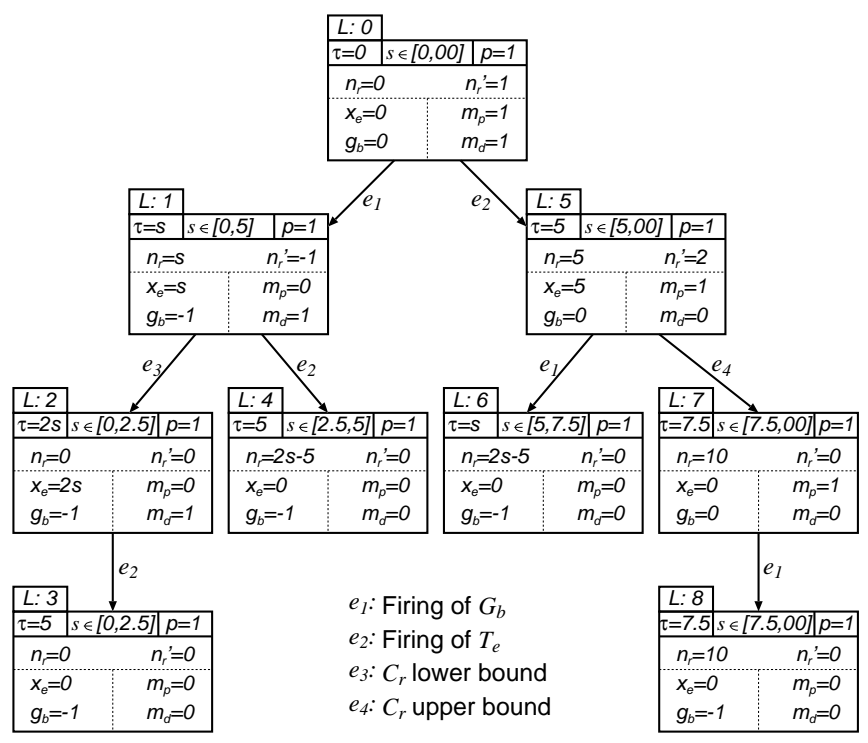

Figure 3. Reachable parametric locations of the reservoir example, starting at time $z=0$

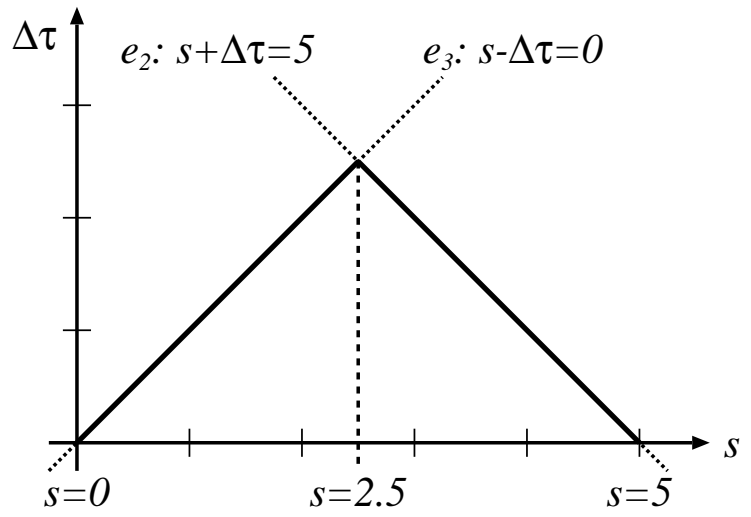

Figure 4. Next minimum event from parametric location 1 depends on value of $s$ 


\section{Measures of Interest}

After the graph of reachable locations has been computed, the probability distribution to be in a state where a predefined condition $\psi$ holds, can be computed by deconditioning the time and the fluid level with the continuous probability density function $g(s)$ that corresponds to the firing time distribution of the general transition. First, the set of successors of a parametric location $L$ for a fixed sample $s$ is defined as:

$$
N(L, s)=\left\{L_{i} \in \operatorname{next}(L) \mid L_{i} . l<s<L_{i} . r\right\},
$$

where next $(L)$ is the set of all successors of location $L$. Then, the minimum firing time of the $s$-successors is given by:

$$
\Gamma(s, \mathrm{~N}(L, s))= \begin{cases}\infty & \mathrm{N}(L, s)=\emptyset \\ L_{i} \cdot \tau(s) & \exists L_{i} \in \mathrm{N}(L, s) .\end{cases}
$$

The function $\Gamma(s, \mathrm{~N}(L, s))$ returns infinity if the set of $s$ successors is empty and the firing time $\tau(s)$ of an arbitrary $s$-successor otherwise. Note that all $s$-successors of $L$ will fire at the same time. A condition $\psi$ can be a conjunction of conditions on the discrete or the fluid marking:

$$
\psi=\psi \wedge \psi\left|\mathbf{m}_{P}=a\right| \mathbf{n}_{C} \leq b,
$$

where $a \in \mathbb{N}, b \in \mathbb{R}^{+}, P \in \mathcal{P}_{D}$ and $C \in \mathbf{P}_{F}$. The function $\xi_{L}^{\psi}(\tau \mid s)$ returns the probability to be in a $\psi$-location at time $\tau$ for a fixed sample $s$ of the general distribution, starting at location $L$ :

$\xi_{L}^{\psi}(\tau \mid s)= \begin{cases}0 & s<L . l \vee s>L . r \\ L . p \cdot I^{\psi}(L) & \Gamma(s, \mathrm{~N}(L, s)) \geq \tau \\ L . p \cdot \sum_{L_{i} \in \mathrm{N}(L, s)} \xi_{L_{i}}^{\psi}(\tau \mid s) & \Gamma(s, \mathrm{~N}(L, s))<\tau\end{cases}$

If sample $s$ lies outside the admissible interval $[l, r]$ of location $L$, we have that $\xi_{L}^{\psi}(\tau \mid s)=0$. If the firing time $\Gamma(s, \mathrm{~N}(L, s))$ is greater or equal than $\tau$, the correct location has been found. Then $L . p \cdot I^{\psi}(L)$ is returned, where $I^{\psi}(L)$ equals 1 if $\psi$ holds in location $L$ and 0 otherwise. In case $\Gamma(s, \mathrm{~N}(L, s))$ is smaller than $\tau$, the correct location has not been found yet and we recurse, by summing the outcome of $\xi_{L_{i}}^{\psi}(\tau \mid s)$ for all $s$-successors $L_{i} \in \mathrm{N}(L, s)$ and multiplying with the probability of the current location L.p.

The probability distribution to be in a $\psi$-location at time $\tau$ is then computed by deconditioning $\xi_{L_{0}}^{\psi}(\tau \mid s)$ according to the probability density function $g(s)$ :

$$
\pi^{\psi}(\tau)=\int \xi_{L_{0}}^{\psi}(\tau \mid s) g(s) d s
$$

Equation (5) can be used for example to compute the probability distribution to have an empty reservoir or to have the pump on or off. It is important to note that the computation method as introduced above allows for the analysis of HPNGs with an arbitrary number of fluid places.

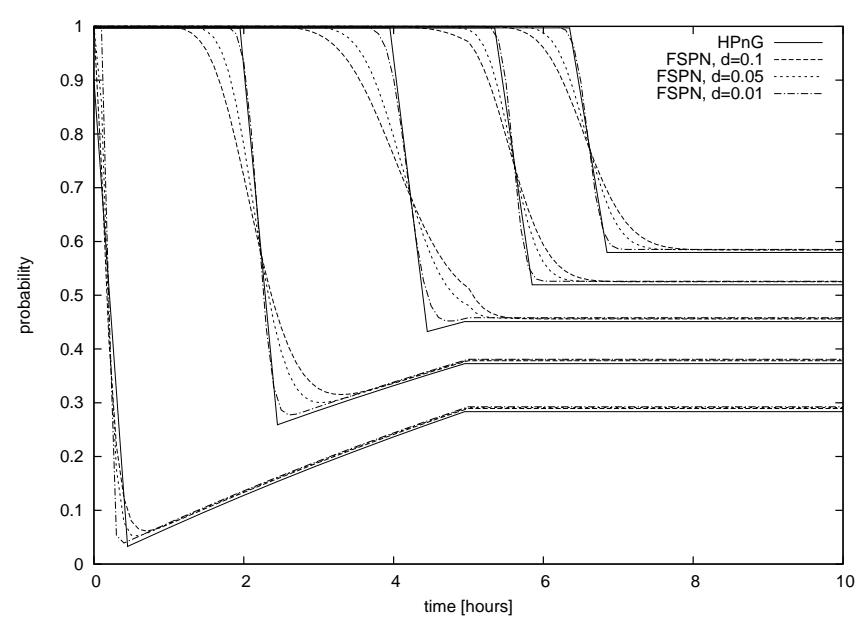

Figure 5. Comparison between the FSPN discretization approach and the HPnG approach with parametric reachability

\section{A. Validation}

We have validated the proposed technique by comparing the results with the ones obtained using another well-known method. In particular, if we consider only one fluid place, and only one exponential transition, the HPnG can be seen as FSPN and can be analyzed using the techniques described in [3]. For a FSPN model, it is possible to automatically derive a set of PDEs (Partial Derivatives Equations). The solution of the PDEs represents the joint probability distribution of having a given fluid level in a given state.

We assign an exponential distribution with mean 7.5 to transition $G_{b}$ in the model proposed in Figure 2. Due to the presence of the deterministic transition $T_{e}$ the model is however not a proper FSPN. Since transition $T_{e}$ is enabled at time $\tau=0$, and fires only once at time $\tau=5$, we can consider two separate models: the first with place $P_{d}$ marked, and the second with place $P_{d}$ empty. Hence, we compute the transient distribution of the first model from time $\tau=0$ until time $\tau=5$. and then use the final state distribution of the first model as the initial state of the second model. The latter is then analyzed for $\tau>5$. Both FSPN models (for $\tau \leq 5$ and for $\tau>5$ ) are analyzed using upwindsemidiscrtization [3].

Figure 5 compares the probability of having less than a given level in the fluid place obtained using the FSPN and the HPnG approaches. As expected, the solution of the FSPN model becomes sharper as the discretization interval $d$ decreases: this is a common property of first-order PDE solution methods such as the upwind-semidiscrtization used in this case. The HPnG solution does not require derivatives approximations and is hence able to produce very sharp results, without depending on a discretization step. Moreover, it is clear from Figure 5 that the FSPN solution tends to the HPnG as $d \leftarrow 0$, which clearly shows that the technique proposed in this paper produces correct results. 


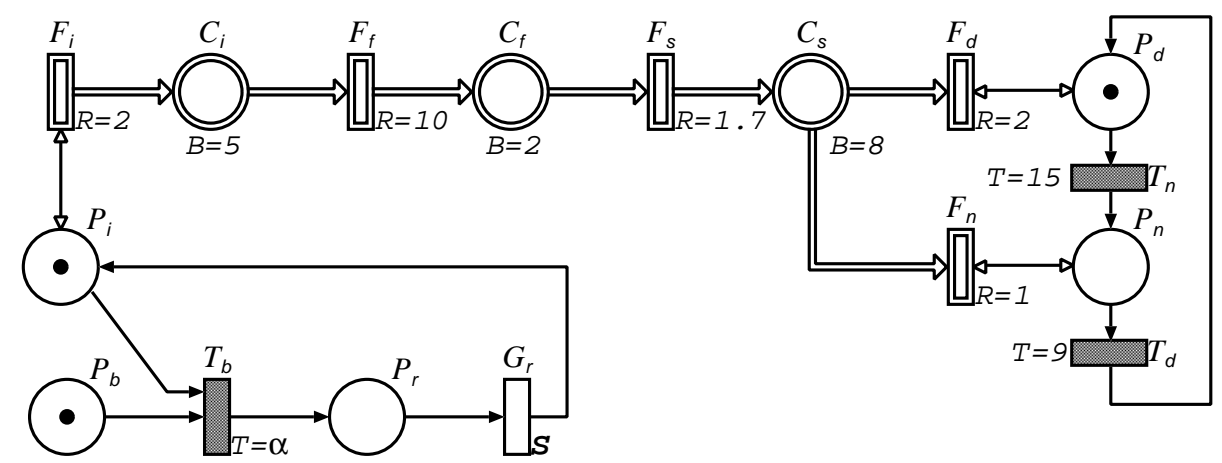

Figure 6. HPnG model of a water cleaning facility

\section{Case Study}

We show the power of the proposed technique by analyzing a simple model of a water treatment facility. The probability distribution of the fluid level in each tank as a function of time is first computed. We then study the probability of having an empty tank as a function of the instant at which the failure occurs. We finally analyze the influence that different repair time distributions (all with the same mean) have on the probability of having an empty tank in the final storage.

Description of the case: Figure 6 presents a hybrid Petri net model of the last two cleaning phases of a water treatment facility, the final water storage and the changing customer demand for nights and days. Fluid place $C_{s}$ represents the final water storage from which the water is distributed to the consumer. The changing demand between day and night is modeled by the two fluid transitions $F_{d}$, firing with rate 2 and $F_{n}$, firing with rate 1 . A token in place $P_{d}$ enables $F_{d}$ via the test arc and a token in $P_{n}$ enables $F_{n}$, respectively. In our model the high demand during the day lasts from 6:00 until 21:00 and the lower demand at night lasts from 21:00 until 6:00, every day. Fluid place $C_{f}$ represents the filtration phase in the water cleaning facility. This process is very fast and has a rather small storage. Fluid place $C_{i}$ represents the softening phase, which is rather slow, but has a larger storage.

The timed transition $T_{b}$ models a failure in the softening phase at specified time $\alpha$. When $T_{b}$ fires, it removes a token from places $P_{b}$ and $P_{i}$ which disables the softening transition $F_{i}$ and places a token in $P_{r}$. This enables the general transition $G_{r}$, which models the repair of the failure by moving the token from $P_{r}$ to $P_{i}$. Recall that the general transition can follow an arbitrary continuous probability distribution. Hence, the model has two free parameters, namely the failure time $\alpha$ and the repair distribution $g(s)$, that are used to analyze the availability of water to the customer in different scenarios. Place $P_{b}$ has been introduced to limit our model to the occurrence of a single fault.
Fluid distribution: First the probability distribution of fluid over time is analyzed for a fixed failure time and repair distribution. The analysis is started at 6:00 in the morning with full storage, filtration and softening tanks. The failure happens at $h=18: 00$ hours and repair follows an exponential distribution with mean 2 hours. Figure 7,8 and 9 show the probability distribution of fluid over time in the places $C_{i}, C_{f}$, and $C_{s}$, respectively. While the x-axis measures time in hours, the $\mathrm{z}$-axis indicates the probability to have less or equal fluid than the bound that is given by the $y-$ axis in $100 \mathrm{~m}^{3}$. Whenever a positive probability to have $\leq 0$ fluid occurs the respective place is empty. In case the final storage is empty, the water demand cannot be served. During the normal operation of the system, the fluid places $C_{i}$ and $C_{f}$ are always completely filled, whereas $C_{s}$ fluctuates due to the different demand during nights and days.

As shown in Figure 7, at time 18:00 hours a failure occurs in the softening phase, breaking the inflow into the softening tank. As fluid is still transferred from the softening tank to the filtration tank, $C_{i}$ is emptied, while the failure continues. This results in a probability 0.23 for the storage tank to be empty at time 21:00 hours. Even though, the repair time is exponentially distributed with mean 2 hours, only at $t=63$ hours, which is 3 days and 1 hour after the failure occurred the failure is repaired with probability 1 (up to the numerical precision of the computation). As soon as the failure is repaired, $C_{i}$ can be refilled. At $t=86$ hours, which is 3 days and 6 hours after the failure occurred, the tank is completely refilled with probability 1 .

Figure 8 shows the effect that the failure in the softening phase has on the fluid distribution in the filtration tank. As can be seen, $C_{f}$ is only affected slightly later and its probability to be empty is much smaller. Recall, that $C_{i}$ becomes empty at 21:00 hours with a positive probability. The filtration tank is rather small and is hence emptied quickly when the water inflow from the softening phase is interrupted. At $t=22.5$ hours, $C_{f}$ is empty with probability 0.11 and at $t=71.5$ hours the tank is fully refilled with probability 1 , again. 
The final storage tank is at its lowest filling due to the deterministic day and night fluctuation every evening at 21:00 hours, which is $350 \mathrm{~m}^{3}$. In case the failure is not repaired before $t=22.5$ hours, the storage does not receive water from the filtration phase any more, and at $t=26.5$ hours the storage itself becomes empty with probability 0.014 . It is interesting to see, that the effect of the failure is propagated to the next evening $(t=45)$, where the probability of the storage to be empty is even higher, namely 0.025 . This is because in normal operation, the storage is refilled during the night to be able to handle the extra demand during the next day. However, the failure at 21:00 slows down the filling of $C_{s}$, resulting in a positive probability of an empty storage the next evening.

Computation times: The algorithms, as discussed in Section III and IV have been implemented in approximately 2000 lines of $\mathrm{C}$-code. The analysis of the model discussed above produces 473 parametric locations for a failure at 18:00 hours. The computation of the parametric locations is done in less than one second, and the deconditioning with an exponential distribution takes 68 seconds on a $2 G h z$ Intel Core 2 Duo processor on a Mac OS X 10.4.11 computer equipped with $1 G B$ of Ram. The integral defined in Equation (5) was computed using the trapezoidal rule and a discretization step $\Delta x=0.005$. The exponential distribution was truncated at $x=45$.

To show the scalability of the approach, we have extended the reservoir example as in Figure 2 by adding (i) more fluid places and fluid transitions between the fluid transitions $F_{p}$ and $F_{d}$, (ii) more fluid and deterministic transitions and discrete places, by duplicating the pump and demand part of the model. Table 1 shows the amount of parametric locations (NPL) and the time for deconditioning (TD) for different numbers of discrete places (NDP), fluid places (NFP), deterministic transitions (NDT) and fluid transitions (NFT). While the computation of the parametric locations is always done in less than one second, the number of parametric locations increases more in NDT than in NFP. This is because fluid places only add more parametric locations in case a boundary is reached while every deterministic transition adds locations upon each firing. The time for deconditioning increases in NFP, as we compute the probability distribution of fluid for every fluid place.

\section{CONCLusions}

The hybrid Petri net formalism with general one-shot transitions (HPNGs) can be used to model systems with an arbitrary number of continuous variables and few stochastic transitions. This makes it useful especially in the context of critical infrastructures, such as a water cleaning facility, where many fluid places are needed to model the different storage tanks. While one-shot transitions are a clear restriction in our modeling formalism, they can be very useful for the dependability analysis of models, where parts fail

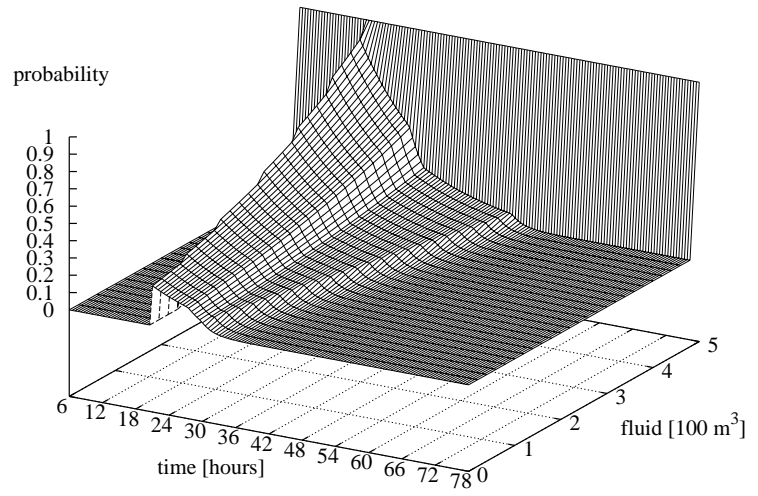

Figure 7. Probability distribution of fluid over time in place $C_{i}$ (softening tank)

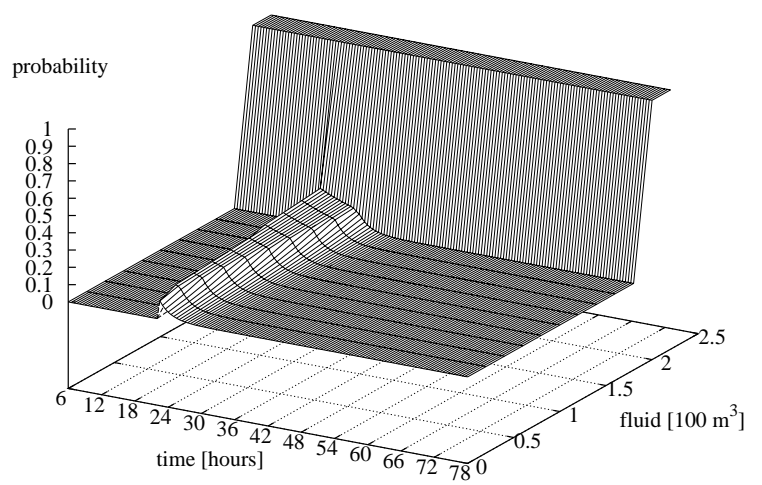

Figure 8. Probability distribution of fluid over time in place $C_{f}$ (filtration tank)

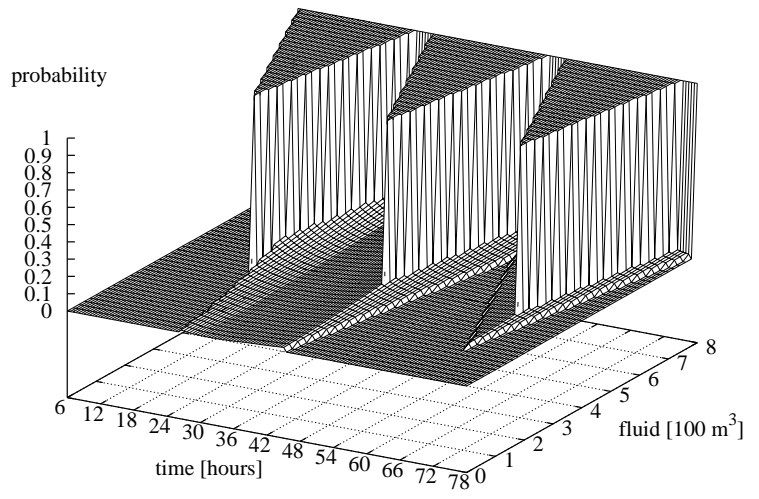

Figure 9. Probability distribution of fluid over time in place $C_{s}$ (storage tank) 


\begin{tabular}{cccccc}
\hline NDP & NFP & NDT & NFT & NPL & TD $(\mathrm{sec})$ \\
\hline 2 & 1 & 1 & 2 & 9 & 3 \\
4 & 3 & 1 & 4 & 13 & 8 \\
6 & 5 & 1 & 6 & 17 & 13 \\
9 & 8 & 1 & 9 & 23 & 20 \\
13 & 12 & 1 & 13 & 31 & 29 \\
\hline 2 & 1 & 1 & 2 & 9 & 3 \\
4 & 1 & 3 & 4 & 20 & 4 \\
6 & 1 & 5 & 6 & 81 & 6 \\
10 & 1 & 9 & 10 & 135 & 11 \\
16 & 1 & 15 & 16 & 258 & 18 \\
\hline
\end{tabular}

Table I

TIME FOR DECONDITIONING (TD) AND NUMBER OF PARAMETRIC LOCATIONS (NPL) FOR DIFFERENT MODELS

and can be repaired up to some maximum number of times. The analysis of HPNGs is done with the efficient concept of Parametric Reachability Analysis, by separating the deterministic and the stochastic evolution of the system. The complexity of this approach is exponential in the number of general transitions, as each firing of a general transition introduces a new random variable for the firing time and hence doubles the number of parametric locations that are reachable without the general transition enabled.

At the moment we can analyze Petri nets with one general transitions that can only fire once. However, we will investigate how the concept can be extended to more general one-shot transitions. This will require a refinement of the algorithm that determines the next event as the segmentation then operates on polyhedra in an n-dimensional space instead of on lines in a two-dimensional space. Clearly this algorithm will have exponential complexity.

Moreover, the approach can be extended to allow for test and inhibitor arcs on fluid places. However, our approach is limited w.r.t. FSPNs and extended hybrid Petri nets as those approaches allow for fluid-dependent flow and transition rates. While the restriction to constant timings and flow rates ensures a linear evolution of the firing of the next possible events over time, incorporating fluid-dependent rates results in non-linear firing times of the next possible events, which the segmentation algorithm cannot handle.

Several performance metrics, such as the distribution of fluid over time, can be derived by deconditioning according to arbitrary continuous probability distributions. The case study clearly shows the feasibility and the scalability of the approach. Future work will improve the deconditioning through a smarter region-based integration approach and will further investigate the complexity and the scalability of the approach.

\section{ACKNOWLEDGEMENTS}

This research has been made possible by a generous funding of the Female Faculty Network of the University of Twente. A. Remke is funded by the 3TU.CeDiCT.

\section{REFERENCES}

[1] F. Sheldon, T. Potok, A. Loebl, A. Frings, and P. Oman, "Managing secure survivable critical infrastructures to avoid vulnerabilities," in Int. Symposium on High Assurance Systems Engineering. IEEE CS Press, 2004, pp. 293-296.

[2] S. Conrad, R. LeClaire, G. O'Reilly, and H. Uzunalioglu, "Critical national infrastructure reliability modeling and analysis," Bell Labs Technical Journal, vol. 11(3), pp. 57-71, 2006.

[3] G. Horton, V. G. Kulkarni, D. M. Nicol, and K. S. Trivedi, "Fluid stochastic Petri nets: theory, application, and solution techniques," European Journal of Operations Research, vol. 105, no. 1, pp. 184-201, Feb. 1998.

[4] M. Gribaudo, M. Sereno, A. Bobbio, and A. Horvath, "Fluid Stochastic Petri Nets augmented with Flush-out arcs: Modelling and Analysis," Discrete Event Dynamic Systems, vol. 11 , no. 1 \& $2,2001$.

[5] K. Wolter, G. Horton, and R. German, "Non-Markovian Fluid Stochastic Petri Nets," Tecnical University of Berlin, Berlin, Germany, Tech. Rep., 1996, report 1996-13.

[6] M. Davis, "Piecewise-deterministic Markov processes: A general class of non- diffusion stochastic models," Journal of the Royal Statistics Society, vol. 46, no. 3, pp. 353-388, 1984.

[7] M. Gribaudo and M. Sereno, "Simulation of Fluid Stochastic Petri Nets," in Proc. of MASCOTS'2000, Aug 2000, pp. 231239.

[8] G. Ciardo, D. M. Nicol, and K. S. Trivedi, "Discrete-event Simulation of Fluid Stochastic Petri Nets," IEEE Transactions on Software Engineering, vol. 2, no. 25, pp. 207-217, 1999.

[9] H. Alla and R. David, "Continuous and Hybrid Petri Nets," Journal of Systems Circuits and Computers, vol. 8, no. 1, Feb 1998.

[10] R. David and H. Alla, "On Hybrid Petri nets," Journal of Discrete Event Dynamic Systems: Theory and Applications, vol. 11, pp. 9-40, 2001.

[11] E. Vicario, L. Sassoli, and L. Carnevali, "Using Stochastic State Classes in Quantitative Evaluation of Dense-Time Reactive Systems," IEEE Transactions on Software Engineering, vol. 99, no. 2, pp. 703-719, 2009.

[12] M. Ajmone Marsan, G. Balbo, G. Conte, S. Donatelli, and G. Franceschinis, Modelling with Generalized Stochastic Petri Nets. John Wiley \& Sons, 1995.

[13] M. Gribaudo and A. Remke, "Hybrid petri nets with general one-shot transitions: model evolution," University of Twente, http://www.vf.utwente.nl/ anne/, Tech. Rep., 2010. 